\title{
Rules for practice in 2008
}

\section{In 2008 I resolve:}

To answer every phone call the same day the call was made. The call may be trivial to me, but it may be important to someone else.

To slow down. I will remember that the patient and his or her relatives are anxious.

Because they are anxious, I will not rush.

I will ask what they want to say, and listen to the answer.

I will not interrupt, even to clarify something.

I will remember that many people are not verbal. They cannot describe what is wrong, but, instead, expect me to somehow know.

I will not scare people with informed consents. I will be reasonable in discussing the risks of surgery. I will say, "in this operation I will be worried about...".

I will recommend surgery only if I am convinced there is a very high chance of helping the patient.

I will surgically change the patient's appearance only if the problem is immediately apparent.

I will decline all minor changes that are seen by the patient only.

I will do only one consultation for one problem at one visit. No more triple consults 'while we are at it'.

I will communicate more by my demeanor and smile, and less with words.

I will make fewer notes while the patient is in the consulting room, and leave my notes for later. I will look at the patient's face when I explain what is wrong. I will use clear, nonmedical language, and repeat it slowly.

I will suggest the safest anesthetic that will allow me to perform the operation properly.

I will assume nothing about what the patient understands.

I will remember the word 'patient' means sufferer, and put myself in the patient's position imagining myself in the other chair looking at me.

I will remove all abruptness and criticism from my tone.

I will make haste slowly. I will try to be on time.

I will refer to colleagues with more expertise than I for a second opinion.

I will write honest referral letters that include my diagnosis.

I will avoid all unnecessary tests, instead relying on my clinical experience in making a diagnosis.

I will tell patients the diagnosis I think is correct and avoid long lists of possibilities.

I will tell patients the one best treatment, the one I recommend, clearly and with confidence. I will explain the second-best treatment and why my first treatment is the best, but I will not confuse by recommending more than two possible treatments.

Even if surrounded by troubled people I resolve to be kind, courteous and patient to all.

I will remember aequanimitas.

I resolve not to talk about myself but rather listen to other people.

I will remember that the world does not revolve around me.

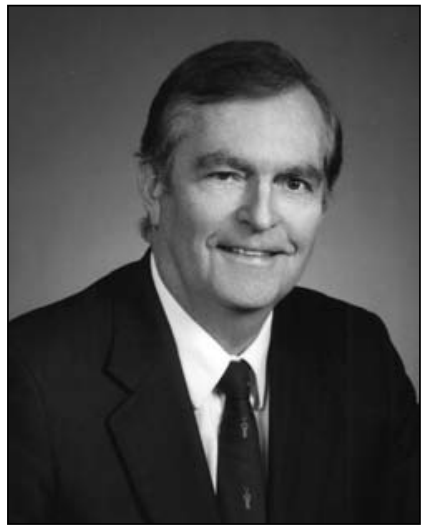

John R Taylor
I resolve not to play the 'can you top this' game. No one wants to know I have had more troubles than they have.

I will not take second-hand gossip or interpreted stories as fact.

I will avoid people who sincerely generalize on the basis of one experience.

I will remember that not all questions are questions. When asked what I think of vitamin E on scars, I will wait until the patient tells me what they already believe. I will not try to change a believer's mind.

I will not ask rhetorical questions in the consulting room. Socrates does not live here anymore.

I will never discuss politics in the consulting room.

I will encourage and give the patient hope.

I will wash my hands before every patient visit and not wear a long tie.

I will rely on my charts, not my memory, in the operating room

I will encourage relatives to attend the consultation, particularly mothers and daughters, and watch their relationship.

I will not overbook. I will learn how to say "no" graciously.

I will telephone referring doctors regarding difficult cases, explaining my advice.

I will be positive every day.

I will enjoy my practice and thank my lucky stars for being able to do this fascinating work. 